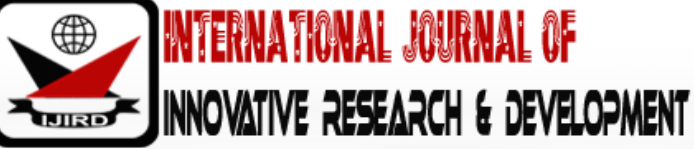

ISSN 2278 - 0211 (Online)

\section{Facility Level Cold-Chain Maintenance, Temperature- Monitoring and Stability of Live Attenuated Vaccines in Kisumu County, Western Kenya}

Moses Olunga Okomo John
Post Graduate Student, Department of Biomedical Sciences,
School of Public Health and Community Development, Kenya
Bernard Guyah PhD
Lecturer, Department of Biomedical Sciences and Technology,
School of Public Health and Community Development, Maseno University, Kenya
Dickens S. Omondi Aduda, PhD
Lecturer, Department of Clinical Medicine, School of Science and Technology,
Kabianga University, Kenya

\begin{abstract}
:
Background: Challenge of live-attenuated vaccines instability in Kenya Expanded Program on Immunization still persists in varying degrees. It potentially affects their safety and clinical performance. Experiences of occasional resurgence of diseases preventable by these vaccines in Kisumu County were suspected to be associated with lapses in cold-chain system at health facility levels. The study assessed cold-chain equipment at facility level to determine specific lapses and their influence on stability of live-attenuated vaccines in Kisumu.

Method: Using a cross-sectional design with repeated analytical observations, site level data was collected in three cycles from a stratified sample of 120 out of 170 health facilities between October 2018 and March 2019. A pretested check list was used. Through SPSS data were summarized descriptively for both dependent and independent variables. Regression with a binomial link performed. Chi-square, Confidence-intervals and odds ratios reported.

Result: Statuses of cold-boxes and vaccines carriers were satisfactory. Of 120 fridges, $60 \%$ were functional; $34.2 \%$ partially; $5.8 \%$ non-functional; $28.3 \%$ expired while $45 \%$ had no sustainable power supply. Private facilities had four of seven non-functional fridges, all being below WHO recommendations. Public facilities were more likely to have stable OPV (95\%C. I 1.4 - 6.7, OR 3.0, p-value 0.006) and measles-rubella (95\% C.I 1.2 - 5.5, OR 2.5, p-value 0.019). Fridges on electric grid without backup were less efficient in maintaining stability of measles-rubella vaccines as compared to fridges on solar (95\%C, I $0.05-0.9$, OR 0.2, p-value 0.0042). Efficiently functional fridges on EG power source with backups significantly increased the likelihood of stable measles-rubella vaccines (95\%C. I 2.4 -187.4 OR 21, p-value 0.006). An expired fridge was less likely to keep stable OPV (95\% C.I 2 - 11, OR 4.7, p-value $\varangle .001)$ and measles-rubella vaccines (95\% C. I $3.1-18.7$, OR 7.1, p-value $<0.001)$. BCG vaccines were unaffected.

Conclusion: Cold-chain management lapses on fridges and occasional erratic power supply, higher at private facilities, significantly destabilize OPV; measles-rubella and ROTA vaccines, so probably associated with upsurges of measles and Rota illnesses. This calls for scrupulous adherence to cold-chain policies specifying scheduled supply / replacement of fridges with power back-up options or solar for sustainability and temperature control.
\end{abstract}

Key words: Electric Grid power source, live attenuated vaccines, fridge tag two and vaccines vials monitors (VVM)

\section{Background}

Vaccines as a technology has significant contribution in improving health indicators in the general population world-wide and more specifically among the under five years (WHO 2015a, 2015c; Angela et al., 2014). Vaccines are fragile; they easily get damaged if exposed to a non-recommended environment. Their stability or potency is only guaranteed if it is able efficiently sensitize body to yield the expected immunity or clinical performance throughout its shelf-life, thus help impact greatly on the reduction of morbidity, mortality and disability due to immunization diseases worldwide. Storage temperature levels determine quality, stability and potency status of each vaccine that in turn, dictates the efficacy and safety of all vaccines over time (WHO 2006, 2015b, Shen, 2014); consequently, loss of vaccine stability contributes to considerable potential for outbreaks of vaccine preventable childhood diseases.

Kenya as a nation being located along equatorial region with very warm climatic situations, require a well thought out, cold-chain system in some specific regions like Kisumu, especially at the peripheral service delivery points which may be more prone to cold chain lapses due to multiple systems and other constrains (WHO2015b). Whereas the Kenya 
government continues to supply immunizing health facilities with new functional fridges, they are not adequately addressing the challenges at hand in that; most of them are designed to only use electricity/ generator but not gas nor solar yet electricity supply in some regions are very erratic; the same fridges hardly form ice packs for outreach use and the quantities are insufficient giving room for sub- standard fridges to be used in private firms. These challenges pose imminent threats to stability of live attenuated vaccines in routine immunization program at Kisumu County in respect to their considered sensitivity level to heat. Cold-chain system in Kisumu was suspected to have challenges with maintaining stability of live attenuated vaccines due to occasional higher resurgence of measles, Rota diarrhoea and endemic nature of tuberculosis, all of which are preventable by live attenuated vaccines. It is worth noting that polio is near eradicated following mass campaigns and introduction of new Inactivated Polio vaccine. The previously reported resurgences were however, inconsistent with the high immunization coverage level, prompting suspicion of a possible breach in cold-chain equipment or sub-optimal systems functioning.

Interestingly, mass immunization campaigns which are often more complex and capital intensive have been linked to better cold-chain management and more efficiency than routine ones, more specifically, the campaigns for measles and polio (MoPHS 2013). This makes it obvious that the inherent challenges are controllable through efficiency enhancement. Identifying established breaches in cold-chain is desirable to strengthen quality vaccines storage at health facility levels. The current study therefore aimed at assessing how maintenance and monitoring of fridges, cold boxes and vaccine carriers influence stability of live attenuated vaccines at health facilities level in Kisumu County. The findings of this study are useful for key stake-holders implementing the Expanded Program On immunization, either as service providers to the end users, supervisors and policy makers at both County and National levels to enable them develop targeted quality improvement measures for relevant aspects.

\section{Methodologies}

\subsection{Design and Sampling Procedures}

Study setting was at immunizing health facilities in the six Sub Counties of Kisumu County. A cross-sectional design with repeated analytical observation was used. Health facilities were categorized based on ownership, either Public or Private/ faith based; their levels of operations as either level two, that is dispensary or level three and above being health centre and all hospitals. A stratified random sampling procedure was then adapted as specified by Yamane (1967 in Israeli 2009). Out of 170 immunizing Health facilities a sample size of 120 fridges, 120 vaccine carriers and 14 cold boxes were obtained, (See Table 1 below) and directly observed for functionality levels, power source, sustainability and expiry status using a pretested quality check list adapted from WHO. Data on equipment effectiveness was obtained from fridge tag 2 gadgets alongside their monitoring charts to a certain temperature trends, while information on stability levels of each vaccine was based on observing stages of vaccine vial monitors (VVM). Whereas stage one and two were considered stable, three or four were classified as unstable and meant for disposal. Data were collected thrice at two months interval between October 2018 and March 2019 to enhance data accuracy and reliability. Cronbach's alpha as a measure of reliability was performed on the three cycles of data and the second cycle reported the highest alpha of 0.8093 . This was treated to be the most reliable, and followed by the first batch with an alpha of 0.7903 and the third with an alpha of 0.7330. (Gliem et al. 2003)

\begin{tabular}{|c|c|c|c|c|c|c|}
\hline Sub County & \multicolumn{2}{|c|}{$\begin{array}{c}\text { Level 3 And } \\
\text { Above } \\
\text { (Hospitals) }\end{array}$} & \multicolumn{2}{c|}{$\begin{array}{c}\text { Level 2 } \\
\text { Health } \\
\text { Facilities }\end{array}$} & $\begin{array}{c}\text { Totals: } \\
\text { Health Facilities And } \\
\text { Samples }\end{array}$ \\
\hline & & GOK & FB / PR & GOK & FB / PR & \\
\hline Kisumu East & Population & 9 & 13 & 14 & 11 & 47 \\
\hline & Sample & 6 & 9 & 10 & 8 & 33 \\
\hline Kisumu West & Population & 3 & 4 & 13 & 3 & 23 \\
\hline & Sample & 2 & 3 & 9 & 2 & 16 \\
\hline Muhoroni & Population & 3 & 2 & 18 & 8 & 31 \\
\hline & Sample & 2 & 1 & 13 & 6 & 22 \\
\hline Seme & Population & 3 & 0 & 21 & 1 & 25 \\
\hline Nyando & Sample & 2 & 0 & 15 & 1 & 18 \\
\hline & Population & 3 & 3 & 14 & & 20 \\
\hline Nyakatch & Sample & 2 & 2 & 10 & 0 & 14 \\
\hline & Population & 4 & 4 & 13 & 3 & 24 \\
\hline Total Health & Sample & 3 & 3 & 9 & 2 & 17 \\
\hline Facilities & & 25 & 26 & 93 & 26 & 120 \\
\hline Sample & & 17 & 18 & 66 & 19 & \\
\hline
\end{tabular}

Table 1: Sampling Procedure and Size

\subsection{Data Analysis}

Data was analyzed using SPSS version 20 and summarized descriptively by frequency charts for the dependent variable (the stability status of live-attenuated vaccines) and tables for effectiveness of independent variables 
(functionality levels, expiry status, types, ownership, source of power and sustainability). The levels of vaccine stability were clustered into two, either "stable" or "unstable" to allow the use of Generalized Estimating Equations algorithm customized for logistic regression with a binomial link. A Chi-square test for independence was performed to detect the association between vaccine stability status and the functionality levels of fridges, cold-boxes and vaccine carriers. Fisher's exact test was used where cell values contained 0 or were below 5 . Binary logistic regression, confidence interval and odds ratios were determined and reported.

\subsection{Limitations}

The study was limited to assessing how fridges, cold-boxes and vaccine carriers influence stability of liveattenuated vaccines at health facilities in Kisumu County, through guidance of Fridge tag 2 and VVM gadgets. It did not include a regional store.

\section{Results}

\subsection{Variable Characteristics}

Health facilities visited in the six sub counties were 120: Kisumu East 33 (27.5\%), Muhoroni 22 (18.32\%), Seme 18 (15.0\%), Nyakatch 17 (14.17\%) Kisumu West $16(13.3 \%)$ and Nyando $14(8.33 \%)$ based on their proportion. In terms of facility type and ownership, there were 80 (66.7\%) public facilities, 40 (33.3\%) private facilities including faith based. As for the level of operation, 84 (70\%) were level two while 36 (30\%) were level 3 and above. Of these, there were 13 public health facilities at level three and above (10.8\%) and 67 at level two (55.8\%). There were 23 private health facilities at level three and above (19.2\%) while $17(14.2 \%)$ at level two. The details are summarized in (Table 2 below).

\begin{tabular}{|c|c|c|c|}
\hline Characteristic & Frequency (\%) & Lower 95\% CI & Upper 95\% CI \\
\hline Type of Facility (Ownership) & & & 76.12 \\
\hline Public & $66.7 \%(n=80)$ & 59.36 & 40.63 \\
\hline Private & $33.3 \%(n=40)$ & 23.88 & \\
\hline Facility Level & & & 73.86 \\
\hline 2 & $70 \%(n=84)$ & 56.78 & 43.21 \\
\hline 3 or higher & $30 \%(n=36)$ & 26.14 & \\
\hline
\end{tabular}

Table 2: Summary Statistics of Personal Characteristics and Facility Information:

Based on VVM staging, $53 \%(n=64)$ of the fridges had stable oral polio vaccines while $47 \%(n=56)$ had some unstable; $70 \%(n=84)$ of the fridges had stable measles-rubella vaccines while $30 \%(n=36)$ had some unstable; $84.2 \%$ $(n=101)$ of the fridges had stable ROTA vaccines while 15.8\% $(n=19)$ had some unstable. Inactivated vaccines were not significantly affected as shown in Figure 1 below.

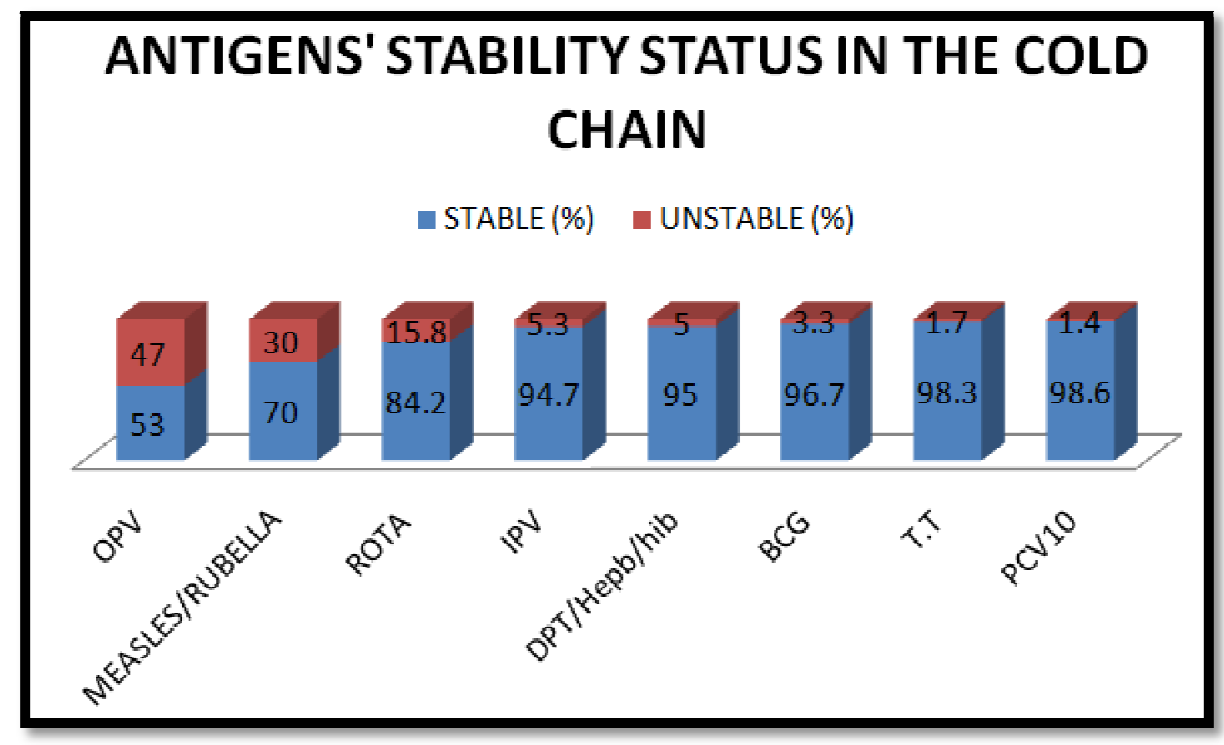

Figure 1: Bar Graphs Showing Proportion Of Fridges versus Stability Status of Each Antigen

\subsection{Influence of Fridges, Cold Boxes and Vaccine Carriers on Stability of Live Attenuated Vaccines.}

\subsubsection{Functionality Levels of Fridges, Cold Boxes and Vaccine Carriers}

Based on fridge tag two gadget assessment during data collection, $60 \%(n=72)$ of fridges were functional, $34.2 \%$ $(n=41)$ were partially functional and $5.8 \%(n=7)$ non-functional. Manufacturers' fridge label indicated that $28.3 \%(n=34)$ of the fridges had expired. Of the 120 vaccine carriers $35.8 \%(n=43)$ were efficiently functional; $61.7 \%(n=74)$ partially functional while $5.8 \%(n=7)$ were non-functional. Up to $55 \%(n=66)$ of the fridges had a sustainable power supply, while the remaining $45 \%(n=52)$ were on electric grid (EG) alone. (See Table 3 below) 


\begin{tabular}{|c|c|c|c|}
\hline Factor & \multicolumn{3}{|c|}{ BATCHES } \\
\hline FRIDGE EXPIRY STATUS & Round 1 & Round 2 & Round 3 \\
\hline $\begin{array}{l}\text { NOT YET } \\
\end{array}$ & $83(69.2)$ & $86(71.7)$ & $89(74.2)$ \\
\hline EXPIRED & $37(30.8)$ & $34(28.3)$ & $31(25.8)$ \\
\hline \multicolumn{4}{|c|}{ FT2 FUNCTIONALITY } \\
\hline YES & $118(98.3)$ & $118(98.3)$ & $117(97.5)$ \\
\hline NO & $2(1.7)$ & $2(1.7)$ & $3(2.5)$ \\
\hline \multicolumn{4}{|c|}{ FRIDGE FUNCTIONALITY } \\
\hline FULLY FUNCTIONAL & $69(57.5)$ & $72(60.0)$ & $74(61.7)$ \\
\hline PARTIALLY FUNCTIONAL & 40 (33.3) & $41(34.2)$ & $39(32.5)$ \\
\hline NON-FUNCTIONAL & $11(9.2)$ & $7(5.8)$ & $5(4.2)$ \\
\hline \multicolumn{4}{|c|}{ COLD BOX FUNCTIONALITY } \\
\hline FULLY FUNCTIONAL & $19(15.8)$ & $2(1.7)$ & $1(0.83)$ \\
\hline PARTIALLY FUNCTIONAL & $2(1.7)$ & $18(15.0)$ & $22(18.37)$ \\
\hline NON-FUNCTIONAL & 99 (82.5) & $1(1.7)$ & $0(0.00)$ \\
\hline NOT APPLICABLE & $0(0.00)$ & $98(81.7)$ & $97(80.8)$ \\
\hline \multicolumn{4}{|c|}{$\begin{array}{l}\text { VACCINE CARRIER FUNCTIONALITY } \\
\end{array}$} \\
\hline EFFEICIENTLY FUNCTIONAL & $42(35.0)$ & $43(35.8)$ & $47(39.2)$ \\
\hline PARTIALLY FUNCTIONAL & $78(65)$ & $74(61.7)$ & $73(60.8)$ \\
\hline NONFUCNTIONAL & $0(0.00)$ & $3(2.5)$ & $0(0.00)$ \\
\hline \multicolumn{4}{|c|}{ POWER SUSTAINABILITY } \\
\hline YES & $72(60.0)$ & $66(55.0)$ & $67(55.8)$ \\
\hline NO & $48(40.0)$ & $54(45.0)$ & $53(44.2)$ \\
\hline \multicolumn{4}{|c|}{ SOURCE OF POWER } \\
\hline ELECTRICITY \& STAND BY GAS & $40(33.3)$ & $29(24.2)$ & $27(22.5)$ \\
\hline ELECTRICITY \& GENERATOR & $21(17.5)$ & $25(20.8)$ & $27(22.5)$ \\
\hline TWO GAS CYLINDER PLUS & $1(0.83)$ & $0(0.00)$ & $0(0.00)$ \\
\hline SOLAR & $11(9.2)$ & $12(10.00)$ & $12(10.00)$ \\
\hline EG. ONLY (NO BACK-UP) & $47(39.2)$ & $54(45.0)$ & $54(45.0)$ \\
\hline \multicolumn{4}{|c|}{ Type of fridge } \\
\hline Domestic & $24(20.0)$ & $21(17.5)$ & $21(17.5)$ \\
\hline MET A WHO & $0(0.00)$ & $2(1.67)$ & $2(1.67)$ \\
\hline RCW2000 DOMETIC & $1(0.83)$ & $1(0.83)$ & $1(0.83)$ \\
\hline TCW3000 DOMETIC & $1(0.83)$ & $1(0.83)$ & $1(0.83)$ \\
\hline RCW42EG DOMETIC & $32(26.7)$ & $27(22.5)$ & $26(21.7)$ \\
\hline RCW50EG DOMETIC & $1(0.83)$ & $1(0.83)$ & $1(0.83)$ \\
\hline SIBIR & $3(2.50)$ & $2(1.67)$ & $2(1.67)$ \\
\hline TCW 15 SDD DOMETIC & $3(2.50)$ & $1(0.83)$ & $1(0.83)$ \\
\hline VEST FROST MK144 & $38(31.7)$ & $39(32.5)$ & $43(35.8)$ \\
\hline VEST FROST VLS024 SDD & $5(4.17)$ & $12(10.0)$ & $11(9.2)$ \\
\hline VEST FROST VLS 054 SDD & $3(2.50)$ & $2(1.67)$ & $2(1.67)$ \\
\hline V.F.VLS 154 SDD & $0(0.00)$ & $2(1.67)$ & $2(1.67)$ \\
\hline VEST FROST VLS 200A.AC & $8(6.70)$ & $8(6.7)$ & $2(1.67)$ \\
\hline VEST FROST VLS 300A.AC & $1(0.83)$ & $1(0.83)$ & $5(4.17)$ \\
\hline
\end{tabular}

Table 3. Functionality Levels of Fridges, Cold-Boxes and Vaccine Carriers in Cold-Chain

\subsubsection{Influences of Fridges, Cold-Boxes and Vaccine Carriers on Stability of Vaccines}

A binary logistic regression with binomial family and logit link was performed in a Generalized Estimating Equations (GEE) algorithm to take care of the three repeated measures observations of the data in this category as shown in Table 4 below. Odds ratios, alongside their p-values at 95\% confidence intervals obtained between the stabilities of antigens against Ownership of Health facilities, expiry status of fridges, fridges functionality levels and source of power supply. The odds ratios were considered significant if their corresponding p-values were less than 0.05 . Fridges supplied with both electricity and gas were 0.225 times less likely to have stable measles-rubella vaccine as compared to fridges on solar (95\% C, I $0.05-0.95$, OR 0.2, p-value 0.0042). A facility owned by government was 3 times more probable to have a stable OPV (95\%C.I $1.4-6.7$, OR 3., p-value 0.006$)$ and 2.5 times highly possible to have a stable measles-rubella (95\%C.I 1.2 - 5.5, OR 2.5, p-value 0.019 ) as compared to one at a private facility. An expired fridge was 4.7 times more predisposed to have an unstable OPV (95\% C.I 1.95 - 11.1, OR 4.7, p-value $\varangle 0.001$ ) and 7.1 times unstable measles-rubella (95\% C.I 3.1 -18.7, OR 7.1, p-value $<0.001$ ) as compared to new. A functional fridge was significant to the stability of all vaccines but only a predictor of measles-rubella (95\%C. I 2.4 -187.4 OR 21, p-value 0.006). 


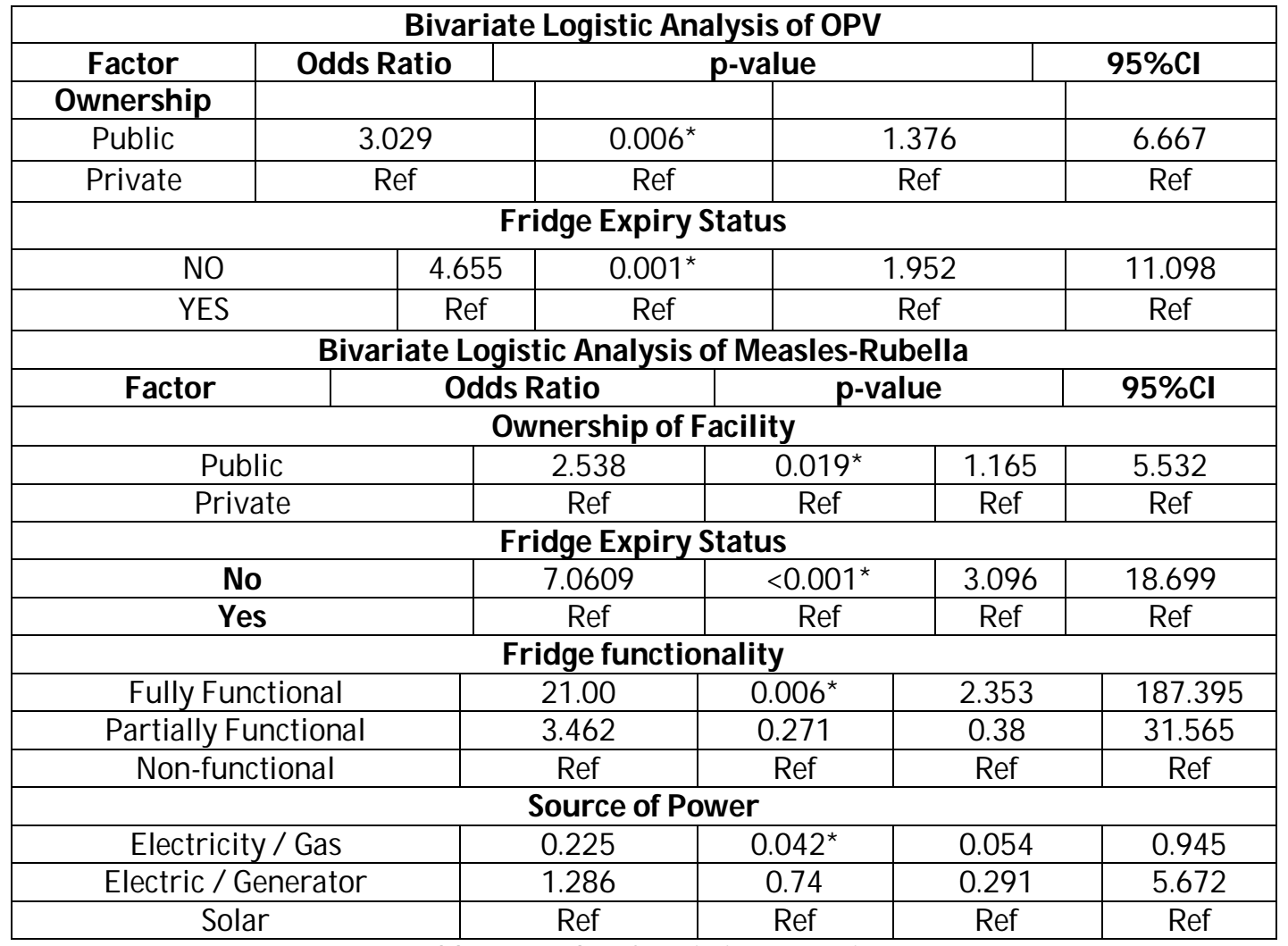

Table 4: Results of Logistic Regression

\section{Discussion}

Whereas it is expected that $100 \%$ of cold-chain equipment should be functional to sustain stability of all vaccines, Kisumu County had $34.2 \%$ of fridges partially functional and $5.8 \%$ non-functional. Non-functional fridges at private facilities were unfortunately below recommendation of WHO. Worse, most of them use EG power source without any backup. Approximately $28.3 \%$ of the fridges in use were expired despite replacement of about $60 \%$ during this study. These contributed significantly to the observed instability of OPV, measles-rubella and Rota vaccines. The study established that statuses of cold boxes and vaccine carriers did not influence stability of vaccines, however, fridges on electric grid (EG) power source without backups (45\%) were less effective in maintaining vaccines stability as compared to solar (most efficient) alongside fridge on EG alternating with gas. Erratic EG power supply in some parts of Kisumu County, predisposed vaccines in the new fridges without backups to risk. The situation was worsened by the fact that generators which might have provided back-ups were only available in about $10 \%$ of these health facilities. Captivatingly, not all these generators were switched on, on time due to unsustainable cost. It appears that Kisumu County is still not in a position to adapt fridges on EG power source alone, but solar driven fridges or EG with gas backups as a realistic well thought of intervention.

The findings show some similarity trends with studies done in other African countries. A study conducted by WHO (2010) in Ethiopia, revealed that 35\% of fridges were not functional, $83 \%$ of the functional equipment were expired and $14 \%$ of the functional equipment were of non-recommended standards for vaccines storage. Both studies share a fact that cold-chain storage logistics at service delivery points in this region have lapses which significantly compromise the stability of live attenuated vaccines. However, the aspect of adapting EG power source without a backup is a new invention whose time is yet to come for Kisumu County. It ultimately impacts negatively on the vaccine performance in diseases control. Sawadago et al (2017) also established 37\% of facilities in lower income countries exposing vaccines to too warm temperatures, though did not specify association to storage logistics.

All the variables established to be of significance to vaccines instability were more prevalent at private facilities. This might be attributed to two facts; one, public health facilities are supplied by government agencies in line with WHO guidelines unlike private institutions with unknown arrangements, and that supervisory roles of the government to inspect and supervise private institutions might have not been observed adequately. The instability trends of antigens indicated that, all live attenuated vaccines apart from BCG were significantly affected by lapses in the cold-chain fridges. It was relatively more stable to higher temperature as compared to OPV, measles-rubella and ROTA vaccines.

\section{Conclusion}

Fridges in cold-chain system at health facilities level in Kisumu County experience cold-chain management lapses that significantly destabilize live attenuated vaccines except for BCG. The prevalence is higher at private health facilities. It is justifiable to conclude that the occasional upsurges of child illnesses such as measles and ROTA diarrhoea in Kisumu County are more closely associated with the observed cold-chain lapses. 


\section{Recommendation}

The lapses in cold-chain system at health facilities level require strict adherence to clear policies which specify scheduled supply / replacement of fridges with power back-up options or solar source for sustainability purposes due to unreliable and erratic electric grid power source in some areas of Kisumu County; focused support supervision by Sub County health management teams (S/ CHMT) and relevant stakeholders requires strengthening for quality interventions and temperature monitoring.

\section{Declarations}

\subsection{Ethics Approval}

Ethical clearance was obtained from Maseno University Ethics Review Committee (Ref: MSU/ DRPI/ MUERC/ 00625/ 18). Consent and administrative approval were also obtained from Kisumu County Director of heath. (Ref: CGK/ DH/ GN/ 133/ VOLII/ (232)/ 18; Medical officers in charges of each of the six Sub Counties and respective in charges / respondents in each health facility sampled.

\subsection{Abbreviations}

BCG: Bacilli Calmette-Guérin, EG: Electric Grid, EPI: Expanded Programme on Immunization, EVSM: Effective vaccine store management, FB: Faith Based, GOK: Government of Kenya, KEPI: Kenya Expanded Programme on Immunization, MOH: Ministry of Health OPV; Oral Polio Vaccine, PCV: Pneumococcal Conjugate Vaccines, PR: Private, S/ CHMT: Sub County Health Management Team, T.T: Tetanus Toxoid vaccine, VVM: Vaccine Vial Monitor, W.H.O: World Health Organization

\subsection{Competing Interest}

The authors declare that they have no competing interests.

\subsection{Authors' contributions}

MOOJ conceived, designed, coordinated and performed the study. MOOJ, BG and DOA analyzed the data. MOOJ and DOA drafted manuscript. All authors read and approved the final manuscript.

\subsection{Disclaimer}

The findings and conclusions presented in this manuscript are those of the authors and do not necessarily reflect the official position of Maseno University

\subsection{Acknowledgements}

We are grateful to all who directly and indirectly participated in this study specifically, the health facilities staff including managers, the Director of health services Kisumu County and all enumerators.

\subsection{Data Sharing}

Requests for the data used for this analysis may be made to lead author, Moses Okomo, jomokomo2010@yahoo.com, and can be shared

\subsection{Funding}

This was a self-sponsored by the lead author who is a $\mathrm{PhD}$ student at Maseno University

\section{Reference}

i. $\quad$ Angela K Shen,a

http:/ / www.ncbi.nlm.nih.gov/ pubmed/ ?term=Fields\%20R\%5BAuthor\%5D\&cauthor=true\&cauthor uid=256114 73Rebecca Fields, ${ }^{b}$ and Mike McQuestionc (Glob Health SciPract. 2014 Dec; 2(4): 381-394. Published online 2014 Dec 2. doi: http:/ / dx.doi.org/ 10.9745\%2FGHSP-D-14-0013710.9745/ GHSP-D-1400137http:/ / dx.doi.org/ 10.9745\%2FGHSP-D-14-00137)The future of routine immunization in the developing world: challenges and opportunities

ii. Gliem, J. A., \& Gliem, R. R. (2003). Calculating, interpreting, and reporting Cronbach's alpha reliability coefficient for Likert-type scales.

iii. Israel, Glenn D. (2009). Sampling the Evidence of Extension Program Impact. Program

iv. Evaluation and Organizational Development, IFAS, University of Florida. PEOD-5. October 2009.

v. Karl Pearson (1895) "Notes on regression and inheritance in the case of two parents,"

vi. $\quad$ Proceedings of the Royal Society of London, 58: 240-242.

vii. MoPHS (2013) Measles vaccine second dose introduction in routine immunization. Guide for health workers. Govt. Printers. Kenya

viii. Ministry of Public Health and Sanitation (2012) EPI operational level manual for service providers. Gvt. Printers. 
ix. Shen. A. K, Fields R, McQuestion M. (2014) the future of routine immunization in the developing world: challenges and opportunities. Glob Health Sci Pact. 2014; 2(4):381-394. http:/ / dx.doi.org/ 10.9745/ GHSP-D-14-00137

x. World Health Organization (2006). Temperature sensitivity of vaccines. Geneva, Switzerland at http:/ / www.who.int/ vaccines-documents/ DocsPDF06/ 847.pdf

xi. World health organization-Unicef (2015a) Challenges in routine immunization in developing countries. Geneva Switzerland.

xii. World Health Organization (2015b). Africa's health monitor. Routine immunization in the WHO African Region: Progress, challenges and way forward: Joseph Okeibunor, e-mail: okeibunorj@who.int

xiii. World Health Organization (2015c) Guidelines on the stability evaluation of vaccines for use under extended controlled temperature conditions. Geniva.

xiv. Yamane, Taro (1967). Statistics, an Introductory Analysis, 2nd Ed., New York: Harper and Row. 